\title{
Optimal asset allocation for aggregated defined benefit pension funds with stochastic interest rates ${ }^{1}$
}

\author{
Ricardo Josa-Fombellida ${ }^{2}$ and Juan Pablo Rincón-Zapatero ${ }^{3}$
}

\begin{abstract}
In this paper we study the optimal management of an aggregated pension fund of defined benefit type, in the presence of a stochastic interest rate. We suppose that the sponsor can invest in a savings account, in a risky stock and in a bond with the aim of minimizing deviations of the unfunded actuarial liability from zero along a finite time horizon. We solve the problem by means of optimal stochastic control techniques and analyze the influence on the optimal solution of some of the parameters involved in the model.
\end{abstract}

Keywords: Pension funds; Stochastic control; Optimal portfolio; Stochastic interest rate

\footnotetext{
${ }^{1}$ Both authors gratefully acknowledge financial support from Consejería de Educación y Cultura de la Junta de Castilla y León (Spain) under projects VA004B08 and VA099/04, and Spanish Ministerio de Ciencia y Tecnología and FEDER funds under project MTM2005-06534. We are particularly thankful to two anonymous referees for many useful comments.

${ }^{2}$ Departamento de Estadística e Investigación Operativa, Universidad de Valladolid, Valladolid, Spain

${ }^{3}$ Departamento de Economía, Universidad Carlos III de Madrid, Madrid, Spain
} 


\section{Introduction}

Pension funds currently represent one of the most important institutions in financial markets because of their high investment capacity and because they complement the role of the Government, allowing those workers who have reached retirement age to maintain their standard of living. These two aspects justify the interest generated over recent years in the study of the optimum management of pension plans.

There are two principal alternatives in pension plan designs with respect to the assignment of risk. In a defined contribution (DC) plan the risk derived from the fund management is borne by the beneficiary. However, in a defined benefit (DB) plan, where the benefits are normally related to the final salary level, the financial risk is assumed by the sponsor agent.

Our aim in this paper is to analyze a BD pension fund of aggregated type, which is a common model in the employment system. We provide here an extension of the previous work of the authors, Josa-Fombellida and Rincón-Zapatero (2001, 2004, 2006, 2008a, 2008b), in an attempt to incorporate more realistic assumptions to the model, dropping the hypothesis of a constant riskless rate of interest. Thus, in our model, there are three sources of uncertainty: i) the fund assets returns; ii) the instantaneous riskless rate of interest; and iii) the evolution of benefits, based on the behavior of salaries and/or other main components of the pension plan.

There are several previous papers dealing with the management of DC funds in the presence of a stochastic rate of interest. Some of them are Boulier et al (2001), Battocchio and Menoncin (2004), Cairns et al (2006) and Menoncin (2005), where the interest rate is assumed to be of the Vasicek type. In Deelstra et al (2003), the interest rate has an affine structure, as in Duffie and Kan (1996) which includes as a special case the CIR and the Vasicek models. Other interesting papers where the interest rate is random, though in a discrete time are Vigna and Haberman (2001) and Haberman and Vigna (2002). The importance of DB funds calls for the completion of the theory studying this case. Moreover, the differences in both types of pension plans makes it impossible to transfer the results from DC to DB plans.

The objective of the shareholder in a DC pension fund is to maximize the expected utility obtained from fund accumulation at a fixed date. The contribution rate is exogenous to this optimization process, since it is generally determined by salary. However, in a 
DB plan the amortization effort is a control variable. The fund assets could be artificially increased with high contributions. Obviously, this makes no sense, since benefits are fixed in advance. Thus, the objective in a DB plan should be related with risk minimization instead of the maximization of fund assets. Of course, the main concern of the sponsor is the solvency risk, related to the security of the pension fund in attaining the comprised liabilities. Similar objectives have been considered in other works, such as Haberman and Sung (1994), Haberman et al (2000) and Josa-Fombellida and Rincón-Zapatero (2001, 2004). The optimal management of DB plans in the presence of a random interest rate is found, but in discrete time, in Haberman and Sung (1994), Chang (1999) and Chang et al (2003).

We make the contribution rate endogenous and dependent on the main variables of the fund, by adopting a spread method of amortization, as in Owadally and Haberman (1999). In this way, the contributions are proportional to the unfunded liabilities, requiring more amortization effort when the plan is underfunded. The pension plan is stochastic, supposing that benefits follow a geometric Brownian motion as in Josa-Fombellida and Rincón-Zapatero (2004). It is then shown that both the stochastic actuarial liability and the normal cost are also geometric Brownian motions, and a relationship between these variables is found. The riskless rate of interest is supposed to be given by a meanreverting process, as in Vasicek (1977). An interesting question addressed in the paper is the selection, according to a valuation criterion, of the technical rate of actualization to value the liabilities. The financial market also comprises a family of zero coupon bonds of fixed maturity and a risky stock, which are correlated with the source of uncertainty of the benefits.

The results obtained are based on the analytical solutions found by means of the dynamic programming approach. The optimal investment in the bond has four summands: i) the classical optimal one in Merton (1971); ii) a positive term decreasing to zero with the terminal date of the plan involving parameters of the riskless rate of interest; iii) the market price of risk multiplied by an expression involving diffusion coefficients of the bond and the stock and the excess expected return of the stock; and iv) a term proportional to the actuarial liability that vanishes if there is no correlation in the financial instruments or if the benefits are deterministic. The optimal investment in the risky asset follows a similar pattern, but in this case there is no corresponding term to those described in i) 
and ii).

The paper is organized as follows. Section 2 defines the elements of the pension scheme of an employment system. We suppose the technical rate of interest is random. The actuarial functions are also introduced and we prove a relation between these functions when the benefits are given by a geometric Brownian motion. In Section 3, we explain the financial market structure. In Section 4, we find a risk-neutral valuation of the liabilities, giving rise to an expression for the technical rate of actualization, that relates it with the interest rate and the correlation parameters between the sources of uncertainty, as well as with the parameters defining the stochastic evolution of liabilities. In Section 5, we consider that the fund is invested in a riskless asset (savings account) and in two risky assets (a bond and a stock). We state the problem of minimizing the expected value of the terminal solvency risk and we explicitly solve it. In Section 6, the results are illustrated with a numerical analysis of the problem, analyzing the investment time evolution pattern in the bond and in the stock. Finally, Section 7 is dedicated to establishing some conclusions and possible extensions. All proofs are in Appendix A.

\section{The pension model}

The pension plan we take into account is an aggregated pension fund of the DB type, thus the benefits are established in advance by the manager. With the objective of the delivery of retirement benefits to the workers, the plan sponsor continuously withdraws time-varying funds. The variables listed below refer to the total group of participants. The principal elements intervening in the funding process and the essential hypotheses allowing its temporary evolution to be determined are as follows. 
Notation of the elements of the pension plan

$T$ : Planning horizon or date of the end of the pension plan, with $0<T<\infty$.

$F(t)$ : Value of fund assets at time $t$.

$P(t)$ : Benefits promised to the participants at time $t$, which are related to the salary at the moment of retirement.

$C(t)$ : Contribution rate made by the sponsor at time $t$ to the funding process.

$A L(t)$ : Actuarial liability at time $t$, that is, total liabilities of the sponsor.

$N C(t)$ : Normal cost at time $t$; if the fund assets match the actuarial liability, and if there are no uncertain elements in the plan, the normal cost is the value of the contributions allowing equality between asset funds and obligations.

$U A L(t)$ : Unfunded actuarial liability at time $t$, equal to $A L(t)-F(t)$.

$M(x) \times 100 \% \quad$ : Percentage of the actuarial value of the future benefits accumulated until age $x \in[a, d]$, where $a$ is the common age of entrance in the fund and $d$ is the common age of retirement for all participants. Function $M$ is a differentiable distribution function on $[a, d]$. In particular, $M(a)=0$ and $M(d)=1$.

$\delta(t)$ : Technical rate of actualization. It is the rate of valuation of the liabilities, which can be specified by the regulatory authorities.

$r(t)$ : Risk-free market interest rate.

Josa-Fombellida and Rincón-Zapatero (2004) considers that there exist disturbances affecting the evolution of the benefits $P$ and hence, the evolution of the normal cost $N C$ and the actuarial liability $A L$, but the rate of valuation $\delta$ of the plan is constant. In this paper we add a more general assumption: we suppose the short rate of interest $r$ is random. This means that $\delta$ is also random. In order to simplify, we will suppose both processes have the same source of uncertainty. As we have commented in the Introduction, three sources of randomness appear in the problem: benefits, interest rate and stock. Thus, to model this situation, we consider a probability space $(\Omega, \mathcal{F}, \mathbb{P})$, where $\mathcal{F}=\left\{\mathcal{F}_{t}\right\}_{t \geq 0}$ is a complete and right continuous filtration generated by the three-dimensional standard Brownian motion, $\mathcal{F}_{t}=\sigma\left\{\left(w(u), w_{B}(u), w_{S}(u)\right): 0 \leq u \leq t\right\}$ and $\mathbb{P}$ is a probability 
measure on $\Omega$. We assume that $r$ and $\delta$ satisfy stochastic differential equations depending on $w_{B}$ only. The benefits randomness is due to another Brownian motion $w_{P}$. Given that the benefits $P$ are conditioned by the increase in salary of the sponsoring employees, we suppose the existence of correlation $q_{1} \in[-1,1]$ between the Brownian motions $w_{P}$ and $w_{B}$, and $q_{2} \in[-1,1]$ between Brownian motions $w_{P}$ and $w_{S}$, which can be explained by the effects of salary on inflation and the effects of the latter on the asset prices. This means that $w_{P}(t)=\sqrt{1-q_{1}^{2}-q_{2}^{2}} w(t)+q_{1} w_{B}(t)+q_{2} w_{S}(t)$, for all $0 \leq t \leq T$, supposing $q_{1}^{2}+q_{2}^{2} \leq 1$. When $q_{1}^{2}+q_{2}^{2}<1$, the risk in the benefits outgo cannot be eliminated by trading in the financial market.

We consider that there $r, \delta$ and $P$ are diffusion processes given by the stochastic differential equations

$$
\begin{aligned}
d r(t) & =\mu_{r}(t, r(t)) d t+\eta_{r}(t, r(t)) d w_{B}(t), \\
d \delta(t) & =\mu_{\delta}(t, \delta(t)) d t+\eta_{\delta}(t, \delta(t)) d w_{B}(t), \\
d P(t) & =\mu_{P}(t, P(t)) d t+\eta_{P}(t, P(t)) d w_{P}(t),
\end{aligned}
$$

for all $t \geq 0$, with $r(0)=r_{0}, \delta(0)=\delta_{0}$ and $P(0)=P_{0}$ representing the initial values of the interest rates and the benefits. However, to obtain a closed form solution we need a more concrete specification of these processes.

We extend the definitions of the actuarial functions from the constant rate of valuation case given in Bowers et al (1986), for deterministic benefits, and Josa-Fombellida and Rincón-Zapatero (2004), for stochastic benefits. The stochastic actuarial liability and the stochastic normal cost are defined as follows:

$$
\begin{aligned}
& A L(t)=\mathbb{E}\left(\int_{a}^{d} e^{-\int_{t}^{t+d-x} \delta(s) d s} M(x) P(t+d-x) d x \mid \mathcal{F}_{t}\right), \\
& N C(t)=\mathbb{E}\left(\int_{a}^{d} e^{-\int_{t}^{t+d-x} \delta(s) d s} M^{\prime}(x) P(t+d-x) d x \mid \mathcal{F}_{t}\right),
\end{aligned}
$$

for every $t \geq 0$, where $\mathbb{E}\left(\cdot \mid \mathcal{F}_{t}\right)$ denotes conditional expectation with respect to the filtration associated to the standard Brownian motion $\left\{\left(w(t), w_{B}(t), w_{S}(t)\right)\right\}_{t \geq 0}$. Thus, to compute the actuarial functions at time $t$, the manager makes use of the information available up to that time, in terms of the conditional expectation. In this way, $A L(t)$ is the total expected value of the promised benefits accumulated according to $M$, discounted at the rate $\delta(t)$, that we suppose is adapted to the filtration. Analogous comments can be given 
to the normal cost $N C(t)$ with function $M^{\prime}$. Note that benefits of retired participants are not a tradable asset and, in consequence, the inherent risk cannot be hedged and the market is incomplete.

Since $P$ is a diffusion process, it satisfies the Markov property (see Øksendal (2003)), hence, conditional expectation with respect to the filtration equals conditional expectation with respect to the current values of $P$ at time $t$. It is plausible to think that, in the task of computing the ideal values of the fund, the information given by the evolution of the random source will be used. Using basic properties of the conditional expectation, the previous definitions can be rewritten:

$$
\begin{aligned}
& A L(t)=\int_{a}^{d} \mathbb{E}\left(e^{-\int_{t}^{t+d-x} \delta(s) d s} P(t+d-x) \mid \mathcal{F}_{t}\right) M(x) d x, \\
& N C(t)=\int_{a}^{d} \mathbb{E}\left(e^{-\int_{t}^{t+d-x} \delta(s) d s} P(t+d-x) \mid \mathcal{F}_{t}\right) M^{\prime}(x) d x .
\end{aligned}
$$

For analytical tractability, we will need a more concrete specification for $P$. A typical way of modelling $P$ in the certain case is to postulate exponential growth, see Bowers et al (1986). The stochastic counterpart is to consider the benefits outgo as a geometric Brownian motion. This is the content of the following hypothesis.

Assumption A. The benefits $P$ satisfies

$$
d P(t)=\mu P(t) d t+\eta P(t) d w_{P}(t), \quad t \geq 0,
$$

where $\mu \in \mathbb{R}$ and $\eta \in \mathbb{R}_{+}$. The initial condition $P(0)=P_{0}$ is a random variable that represents the initial liabilities.

Hence, we are supposing that the benefits increase or decrease on average at a constant exponential rate. The behavior of the actuarial functions $A L$ and $N C$ is then given in the following proposition. To this end, we define the following random functions:

$$
\begin{aligned}
& \psi_{A L}(t)=\int_{a}^{d} e^{\int_{t}^{t+d-x}(\mu-\delta(s)) d s} M(x) d x \\
& \psi_{N C}(t)=\int_{a}^{d} e^{\int_{t}^{t+d-x}(\mu-\delta(s)) d s} M^{\prime}(x) d x \\
& \xi_{A L}(t)=\int_{a}^{d} e^{\int_{t}^{t+d-x}(\mu-\delta(s)) d s}(\mu-\delta(t+d-x)) M(x) d x-(\mu-\delta(t)) \psi_{A L}(t) .
\end{aligned}
$$


Proposition 2.1 Under Assumption A the actuarial functions satisfy $A L=\psi_{A L} P$ and $N C=\psi_{N C} P$, and they are linked by the identity

$$
\left(\mu+\frac{\xi_{A L}(t)}{\psi_{A L}(t)}\right) A L(t)+N C(t)-P(t)=0
$$

for every $t \geq 0$. Moreover, the actuarial liability satisfies

$$
d A L(t)=\left(\mu+\frac{\xi_{A L}(t)}{\psi_{A L}(t)}\right) A L(t) d t+\eta A L(t) d w_{P}(t), \quad A L(0)=A L_{0}=\psi_{A L}(0) P_{0} .
$$

Thus, $A L$ is a geometric Brownian motion with random drift. Processes $A L$ and $P$ differ in the drift term $\left(\xi_{A L}(t) / \psi_{A L}(t)\right) A L$. Notice that $\psi_{A L}(t)$ can be interpreted as the discounted value, at rate $\delta(s)-\mu$, of a security paying an amount of $M(x)$ continuously in the interval $[t, t+d-a]$. Since $\xi_{A L}(t)$ is the derivative of $\psi_{A L}(t)$, the quotient $\xi_{A L}(t) / \psi_{A L}(t)$ is the stochastic rate of growth of the above discounted value. Thus, the drift of $d A L$ given in (2) takes into account not only the mean growth of the liabilities, $\mu$, but the random fluctuations due to the stochastic $\delta$.

We will now use a spread method of fund amortization, as mentioned in the Introduction. Thus, we will assume that the supplementary contribution rate (difference between contribution rate and normal cost) is proportional to the unfunded actuarial liability, that is

$$
C(t)=N C(t)+k(A L(t)-F(t)),
$$

where $k$ is a constant selected by the employer, representing the rate at which surplus or deficit is amortized. Though actuarial practice takes $1 / k$ equal to a continuous annuity with amortization over $m$ years, we consider more flexibility in the selection of $k$ than actuarial practice suggests, as in Haberman and Sung (1994) or Josa-Fombellida and Rincón-Zapatero (2001, 2004).

\section{The financial market}

In this section we describe the underlying financial market in our model. The plan sponsor manages the fund by means of a portfolio formed by a riskless $R$, a coupon zero bond $B$ and a stock $S$.

First we assume the following hypothesis. 
Assumption $\mathbf{B}$. The instantaneous riskless interest rate $r(t)$ satisfies the stochastic differential equation:

$$
d r(t)=\alpha(\beta-r(t)) d t+\sigma d w_{B}(t), \quad r(0)=r_{0},
$$

where $\alpha, \beta$ and $\sigma$ are strictly positive constants.

This process of type mean-reverting and known as the Orstein-Uhlenbeck process, has been introduced in Vaşicek (1977) to explain interest rate behavior.

We assume the price process of the riskless asset $R$ is given by

$$
d R(t)=r(t) R(t) d t, \quad R(0)=R_{0},
$$

where the evolution of $r(t)$ is given by (4). This asset can be interpreted as a bank account paying the instantaneous interest rate $r(t)$ without any risk.

Given $r$ we assume that there exists a market for zero coupon bonds with a fixed maturity $T_{1}>T$. Following Vasicek (1977) (see also Battocchio and Menoncin (2004)) the price at instant $t$ of a zero coupon bond with maturity $T_{1}$, with $t<T<T_{1}$, is given by

$$
B\left(t, T_{1}\right)=e^{c\left(t, T_{1}\right)-b\left(t, T_{1}\right) r(t)},
$$

where

$$
\begin{aligned}
& b\left(t, T_{1}\right)=\frac{1}{\alpha}\left(1-e^{-\alpha\left(T_{1}-t\right)}\right), \\
& c\left(t, T_{1}\right)=-R(\infty)\left(T_{1}-t\right)+b\left(t, T_{1}\right)\left(R(\infty)-\frac{\sigma^{2}}{2 \alpha^{2}}\right)+\frac{\sigma^{2}}{4 \alpha^{3}}\left(1-e^{-2 \alpha\left(T_{1}-t\right)}\right),
\end{aligned}
$$

and $R(\infty)=\beta+\sigma \zeta \alpha-\sigma^{2} /\left(2 \alpha^{2}\right)$ represents the return of a zero coupon bond with maturity equal to infinite, and $\zeta$ is the constant market price of risk. Applying Itô's formula, ${ }^{4}$ the price of the bond process verifies the stochastic differential equation

$$
d B\left(t, T_{1}\right)=B\left(t, T_{1}\right)\left(\left(r(t)+\sigma \zeta b\left(t, T_{1}\right)\right) d t-\sigma b\left(t, T_{1}\right) d w_{B}(t)\right), \quad B\left(T_{1}, T_{1}\right)=0 .
$$

\footnotetext{
${ }^{4}$ Given an scalar Itô process $d X(t)=\mu(t, X(t)) d t+\sigma(t, X(t)) d W(t)$ with $W$ a standard Brownian motion, and a function $f$ that is twice continuously differentiable on $[0, \infty) \times \mathbb{R}, Y(t)=f(t, X(t))$ is again an Itô process and (Itô's formula)

$d Y(t)=\left(f_{t}(t, X(t))+f_{x}(t, X(t)) \mu(t, X(t))+\frac{1}{2} f_{x x}(t, X(t)) \sigma^{2}(t, X(t))\right) d t+f_{x}(t, X(t)) \sigma(t, X(t)) d W(t)$.
} 
Finally, we consider a stock whose dynamic is given by the stochastic differential equation

$$
d S(t)=S(t)\left(\mu_{S}(r(t)) d t+\sigma_{r} d w_{B}(t)+\sigma_{S} d w_{S}(t)\right), \quad S(0)=s_{0}
$$

where $\sigma_{r}, \sigma_{S}$ are positive constants defining the stock volatility, that is $\sqrt{\sigma_{r}^{2}+\sigma_{S}^{2}}$, and the drift parameter $\mu_{S}(r)$ is the instantaneous mean having the form $\mu_{S}(r)=r+m_{S}$, with $m_{S}$ a constant representing the expected excess return from investing in the stock, as in Deelstra et al (2003), Battocchio and Menoncin (2004) or Menoncin (2005).

\section{Risk neutral valuation of the liabilities}

The accurate valuation of liabilities is of the utmost importance for the sponsor. We address here the question of how to perform a fair valuation, keeping in mind that the promised benefits to participants is not a tradeable asset. To circumvent this problem we resort to a valuation based on a concept of equilibrium, see e.g. Constantinides (1978). The risk-neutral valuation of liabilities offers a univocally defined value for the technical rate of actualization, $\delta$. This value is a modification of the short rate of interest, $r$, to take into account the drift and diffusion components of the financial instrument and benefits as well as the several correlations existing between them.

For age $x$, let $Y^{x}(t, P)$ be the asset, valued at time $t$, consisting in a payment of $P$ monetary units at the age of retirement, $d$, to a participant with current age $x$. Process $P$ is a geometric Brownian motion according to Assumption A. As $P$ is not tradeable, it cannot be used to form a portfolio to hedge the risk. Thus we form a portfolio formed by $Y^{x}, B$ and $S$, with two tradeable assets and three independent Brownian motions. To obtain a risk neutral valuation it is assumed that the risk uncorrelated with the two freely traded financial instruments, i.e. the stock and the bond, is not priced. Notice that the actuarial liability of the fund is

$$
A L(t)=\int_{a}^{d} Y^{x}(t, P(t)) M(x) d x .
$$

Once $Y^{x}$ is found, and after matching this expression of $A L$ with that given in Section 2 , the value of $\delta$ is determined. To this end, consider the asset at any intermediate time, $Y^{x}(t+\tau, P)$, for $0 \leq \tau \leq d-x$. Forming a portfolio $\Pi=Y^{x}+\pi_{B} B+\pi_{S} S$ with one unit 
of asset $Y^{x}, \pi_{B}$ units of $B$ and $\pi_{S}$ units of $S$, and applying Itô's formula-see footnote 4-, we have

$$
\begin{aligned}
d \Pi= & d Y^{x}+\pi_{B} d B+\pi_{S} d S \\
= & \left(Y_{p}^{x} \mu P+\frac{1}{2} Y_{p p}^{x} \eta^{2} P^{2}+Y_{\tau}^{x}\right) d t+Y_{p}^{x} \eta P d w_{P} \\
& +\pi_{B}\left((r+\sigma \zeta b) B d t-\sigma b B d w_{B}\right)+\pi_{S}\left(\mu_{S}(r) S d t+\sigma_{r} S d w_{B}+\sigma_{S} S d w_{S}\right) \\
= & \left(Y_{p}^{x} \mu P+\frac{1}{2} Y_{p p}^{x} \eta^{2} P^{2}+Y_{\tau}^{x}+\pi_{B}(r+\sigma \zeta b) B+\pi_{S} \mu_{S}(r) S\right) d t \\
& +Y_{p}^{x} \eta \sqrt{1-q_{1}^{2}-q_{2}^{2}} P d w+\left(Y_{p}^{x} \eta P q_{1}-\pi_{B} \sigma b B+\pi_{S} \sigma_{r} S\right) d w_{B}+\left(Y_{p}^{x} \eta P q_{2}+\pi_{S} \sigma_{S} S\right) d w_{S} .
\end{aligned}
$$

The first equality is due to the self-financing property of the strategies $\pi_{B}, \pi_{S}$. In the following equalities we use Assumption A, (6) and (7).

Now we select $\pi_{B}$ and $\pi_{S}$ in order to eliminate the risks related with $w_{B}$ and $w_{S}$, that is $\pi_{B} B=\left(Y_{p}^{x} \eta P q_{1}+\pi_{S} \sigma_{r} S\right) /(\sigma b)$ and $\pi_{S} S=-Y_{p}^{x} \eta P q_{2} / \sigma_{S}$. We also disregard the risk orthogonal to them, that is, the risk related with $w$ is not priced. The total return of the hedge portfolio must be equal to the rate of interest at time $t+\tau, r(t+\tau)$. Thus, we obtain

$$
r(t+\tau)\left(Y^{x}+\pi_{B} B+\pi_{S} S\right)=Y_{\tau}^{x}+Y_{p}^{x} \mu P+\frac{1}{2} Y_{p p}^{x} \eta^{2} P^{2}+\pi_{B}(r+\sigma \zeta b) B+\pi_{S} \mu_{S}(r) S,
$$

that, with the expressions for $\pi_{B}$ and $\pi_{S}$ found above and using $\mu_{S}(r)=r+m_{S}$, becomes the pricing partial differential equation

$$
r(t+\tau) Y^{x}=Y_{\tau}^{x}+\omega P Y_{p}^{x}+\frac{1}{2} \eta^{2} P^{2} Y_{p p}^{x}
$$

with boundary conditions $Y^{x}(t+d-x, P)=P, Y^{x}(t+\tau, 0)=0$, and where

$$
\omega=\mu+\zeta \eta q_{1}-\frac{m_{S}+\zeta \sigma_{r}}{\sigma_{S}} \eta q_{2}
$$

The solution to $(8)$ is $Y^{x}(t+\tau, P)=P e^{-\int_{t+\tau}^{t+d-x}(r(s)-\omega) d s}$ hence, $Y^{x}(t, P)=P e^{-\int_{t}^{t+d-x}(r(s)-\omega) d s}$. Consequently,

$$
A L(t)=\int_{a}^{d} Y^{x}(t, P(t)) M(x) d x=P(t) \int_{a}^{d} e^{-\int_{t}^{t+d-x}(r(s)-\omega) d s} M(x) d x .
$$

On the other hand, by Proposition 2.1 the actuarial liability satisfies

$$
A L(t)=P(t) \psi_{A L}(t)=P(t) \int_{a}^{d} e^{\int_{t}^{t+d-x}(\mu-\delta(s)) d s} M(x) d x .
$$

Comparing both values of $A L, \delta(t)$ must be chosen equal to $r(t)+\mu-\omega$ in order to attain a risk-neutral valuation. 
Thus we will assume throughout the paper, in a similar way to that found in JosaFombellida and Rincón-Zapatero (2004, 2008b), that the technical interest rate coincides with the rate of return of the bond modified to get rid of the sources of uncertainty, that is:

Assumption C. The technical rate of actualization is chosen to be

$$
\delta(t)=r(t)-\zeta \eta q_{1}+\frac{m_{S}+\zeta \sigma_{r}}{\sigma_{S}} \eta q_{2}
$$

Besides the risk-neutral valuation it provides, this selection of $\delta$ allows us to solve explicitly the problem in the following section.

\section{The optimal portfolio}

In this section we analyze how the sponsor may select, in an optimal way, the proportion of fund assets put into a savings account, or invested in a bond and in a risky stock. Thus, the sponsor faces three elements of randomness: one due to the benefits, which is inherent to the pension plan, and the other two are financial market variables, specifically the stochastic interest rate and a risky stock.

The plan sponsor invests the fund in a portfolio formed by the savings account $R$, given by (5), the zero-coupon bond $B$ with maturity $T_{1}>T$, given by (6), and the stock $S$ whose dynamic is given by (7).

The amounts invested in the bond $B$ and the stock $S$ are denoted by $\lambda_{B}$ and $\lambda_{S}$, respectively. The remainder, $F-\lambda_{B}-\lambda_{S}$, goes to the savings account $R$. Borrowing and shortselling is allowed. A negative value of $\lambda_{B}\left(\right.$ resp. $\left.\lambda_{S}\right)$ means that the sponsor sells shares of $B$ (resp. S) short, while, if $\lambda_{B}+\lambda_{S}$ is greater than $F$, then he or she gets into debt to purchase the stocks, borrowing money at the interest rate $r$.

We suppose $\left\{\left(\lambda_{B}(t), \lambda_{S}(t)\right): t \geq 0\right\}$ is a Markovian control process adapted to filtration $\left\{\mathcal{F}_{t}\right\}_{t \geq 0}$ and satisfying

$$
\mathbb{E} \int_{0}^{T}\left(\lambda_{B}^{2}(t)+\lambda_{S}^{2}(t)\right) d t<\infty .
$$

Therefore, the fund's dynamic evolution under the investment policy $\left(\lambda_{B}, \lambda_{S}\right)$ is:

$$
d F(t)=\lambda_{B}(t) \frac{d B(t)}{B(t)}+\lambda_{S}(t) \frac{d S(t)}{S(t)}+\left(F(t)-\lambda_{B}(t)-\lambda_{S}(t)\right) \frac{d R(t)}{R(t)}+(C(t)-P(t)) d t
$$


By substituting (5), (6) and (7) in (10), and taking into account Proposition 2.1 and (3), we obtain:

$$
\begin{aligned}
d F(t) & =\left(b(t) \sigma \zeta \lambda_{B}(t)+\lambda_{S}(t)\left(\mu_{S}(r(t))-r(t)\right)+(r(t)-k) F(t)\right. \\
& \left.+\left(k+\mu+\frac{\xi_{A L}(t)}{\psi_{A L}(t)}-\delta(t)\right) A L(t)\right) d t \\
& +\left(-b(t) \sigma \lambda_{B}(t)+\sigma_{r} \lambda_{S}(t)\right) d w_{B}(t)+\sigma_{S} \lambda_{S}(t) d w_{S}(t),
\end{aligned}
$$

with initial condition $F(0)=F_{0}>0$. By $(2)$, and the relation $w_{p}=\sqrt{1-q_{1}^{2}-q_{2}^{2}} w+$ $q_{1} w_{B}+q_{2} w_{S}$ in terms of $X=F-A L$ equation (11) is

$$
\begin{aligned}
d X(t)= & \left(b(t) \sigma \zeta \lambda_{B}(t)+m_{S} \lambda_{S}(t)+(r(t)-k) X(t)+(r(t)-\delta(t)) A L(t)\right) d t \\
& -\eta \sqrt{1-q_{1}^{2}-q_{2}^{2}} A L(t) d w(t)-\left(b(t) \sigma \lambda_{B}(t)-\sigma_{r} \lambda_{S}(t)+\eta q_{1} A L(t)\right) d w_{B}(t) \\
& +\left(\sigma_{S} \lambda_{S}(t)-\eta q_{2} A L(t)\right) d w_{S}(t),
\end{aligned}
$$

with the initial condition $X(0)=X_{0}$.

Let us now turn to the preferences of the controller. We assume that he or she wishes to minimize the terminal solvency risk. Thus, the objective functional to be minimized over the class of admissible controls $\mathcal{A}_{X_{0}, A L_{0}, r_{0}}$, is given by

$$
J\left(\left(X_{0}, A L_{0}, r_{0}\right) ;\left(\lambda_{B}, \lambda_{S}\right)\right)=\mathbb{E}_{X_{0}, A L_{0}, r_{0}} X^{2}(T) .
$$

Here, $\mathcal{A}_{X_{0}, A L_{0}, r_{0}}$ is the set of measurable processes $\left\{\left(\lambda_{B}(t), \lambda_{S}(t)\right)\right\}_{t \geq 0}$ where $\left(\lambda_{B}, \lambda_{S}\right)$ satisfies (9) and where $X, A L$ and $r$ satisfy (12), (2) and (4), respectively. In the above, $\mathbb{E}_{X_{0}, A L_{0}, r_{0}}$ denotes conditional expectation with respect to the initial conditions $\left(X_{0}, A L_{0}, r_{0}\right)$.

In the following developments we will suppose only the underfunded case where $X_{0}<$ 0 , so we will refer to $X$ as debt. The overfunded case, $X_{0}>0$, leads to similar results.

The dynamic programming approach is used to solve the problem. To make the process work, some properties of the value function need to be established. The value function is defined as

$$
\widehat{V}(t, X, A L, r)=\min _{\left(\lambda_{B}, \lambda_{S}\right) \in \mathcal{A}_{t, X}, A L, r}\left\{J\left(t,(X, A L, r) ;\left(\lambda_{B}, \lambda_{S}\right)\right): \text { subject to }(12),(2),(4)\right\} .
$$


The connection between value functions in optimal control theory (deterministic or stochastic) and optimal feedback controls is accomplished by the HJB equation, see Fleming and Soner (1993).

We have the following result.

Theorem 5.1 Suppose that Assumptions A, B and C hold. Then the optimal investments are given by

$$
\begin{aligned}
& \lambda_{B}^{*}(t, X, A L)= \frac{-\alpha}{\sigma\left(1-e^{-\alpha\left(T_{1}-t\right)}\right)}\left(\left(\zeta-2 \frac{\sigma}{\alpha}\left(1-e^{-\alpha(T-t)}\right)+\frac{m_{S} \sigma_{r}+\zeta \sigma_{r}^{2}}{\sigma_{S}^{2}}\right) X\right. \\
&\left.+\left(q_{1}-\frac{\sigma_{r}}{\sigma_{S}} q_{2}\right) \eta A L\right) \\
& \lambda_{S}^{*}(t, X, A L)=-\frac{m_{S}+\zeta \sigma_{r}}{\sigma_{S}^{2}} X+\frac{q_{2}}{\sigma_{S}} \eta A L .
\end{aligned}
$$

Remark 5.1 From (15) in Theorem 5.1, the optimal investments do not depend on $r$ and the investment in the bond is of the form

$$
\begin{aligned}
\lambda_{B}^{*}(t, X, A L)= & \frac{1}{1-e^{-\alpha\left(T_{1}-t\right)}}\left(\frac{-\alpha \zeta}{\sigma} X+2\left(1-e^{-\alpha(T-t)}\right) X\right. \\
& \left.-\frac{\alpha}{\sigma \sigma_{S}^{2}}\left(m_{S} \sigma_{r}+\zeta \sigma_{r}^{2}\right) X-\frac{\alpha \eta}{\sigma \sigma_{S}}\left(\sigma_{S} q_{1}-\sigma_{r} q_{2}\right) A L\right) .
\end{aligned}
$$

Leaving aside the common factor, it is the sum of four terms. The first term coincides with the classical optimal one in Merton (1971) when the coefficients are deterministic. The second term is proportional to $X$ and depends on the time horizon planned, vanishing at the terminal date $T$. The third term is also proportional to the debt, with a coefficient that depends on several of the elements defining the prices of the bond and the stock. The fourth term is quite different, as it involves random liability instead of debt. The summand is now proportional to $A L$, with a coefficient that depends on the volatilities of the processes $A L, B$ and $S$ and their respective correlations. Thus, this last term cares about the random evolution of liabilities. In fact it vanishes when benefits are deterministic or when the relation: $q_{1} \sigma_{S}=q_{2} \sigma_{r}$, between variances and covariances holds. In both cases the optimal investment in the bond is proportional to the unfunded liability, $U A L=-X$. 
The optimal investment in the stock given by (16) is simpler. It is the sum of two terms, one proportional to debt $X$ and the other proportional to the actuarial liability $A L$. The latter is zero, that is, $\lambda_{S}^{*}$ is proportional to debt $X$, if either the benefits are deterministic or when there is no correlation between stock and benefits.

Substituting (15) and (16) in (12) we obtain that the optimal debt satisfies the stochastic differential equation:

$$
\begin{aligned}
d X(t)= & \left(-\zeta^{2}-\frac{\left(m_{S}+\zeta \sigma_{r}\right)^{2}}{\sigma_{S}^{2}}+\frac{2}{\alpha}\left(1-e^{-\alpha(T-t)}\right) \zeta \sigma+r(t)-k\right) X(t) d t \\
& -\eta \sqrt{1-q_{1}^{2}-q_{2}^{2}} A L(t) d w(t)+\left(\zeta-\frac{2}{\alpha}\left(1-e^{-\alpha(T-t)}\right) \sigma\right) X(t) d w_{B}(t) \\
& -\frac{m_{S}+\zeta \sigma_{r}}{\sigma_{S}} X(t) d w_{S}(t)
\end{aligned}
$$

with the initial condition $X(0)=X_{0}$ and where $A L$ is given by (2) and $r$ by (4). In the following section we will numerically integrate the linear system of SDEs formed by (2), (4) and (17) to illustrate the results.

\section{A numerical illustration}

In this section we consider a numerical application in order to illustrate the dynamic behavior of the debt and its expected value, and the optimal portfolio strategy. The parameters defining the financial market have been taken from Boulier et al (2001). Thus, the initial value for the interest rate $r_{0}=0.05$ coincides with its equilibrium value $\beta$, the maturity is $T_{1}=10$ and the market price of risk is $\zeta=0.15$. These and the remainder parameter values are shown in Table 1.

\section{[INSERT TABLE 1 HERE]}

We consider a contribution period before retirement of $T=6$ years and that benefits are random with $\mu=0.04$ and $\eta=0.08$. The effort of amortization is $k=0.06$. The initial values for the actuarial liability and the fund wealth are taken to be $A L_{0}=100$ and $F_{0}=80$ respectively, so $X_{0}=-20$, that is the fund is $20 \%$ underfunded. Initial 
benefits are supposed to be $1 \%$ of $A L_{0}$, that is, $P_{0}=1$. It is supposed that benefits are accumulated uniformly, $M(x)=(x-a) /(d-a)$.

The correlation between benefits and short rate is selected as $q_{1}=0.2$ and the correlation between benefits and stock as $q_{2}=0.2$. Figure 1 shows the evolution of debt, fund assets, actuarial liability and its expected values along the planning interval. First, we have run a sample path of $r$ in the interval $[0, T+d-a]$, which is needed to obtain $\psi_{A L}(t)$ and $\xi_{A L}(t)$ for $t \in[0, T]$. To obtain $r$, we have used the Euler method, see e.g. Kloeden and Platen $(1999)^{5}$, and to obtain both $\psi_{A L}(t)$ and $\xi_{A L}(t)$, the composed trapezoidal rule has been used to compute the integrals. With this data at hand, the Euler scheme has been employed again to find the solution of the system (2), (4) and (17), of course with the same sample path used for $r$, restricted to $[0, T]$. This particular sample path drawn in Figure 1 shows that the debt takes values in the range -43 to -12 and it attains the value of -16.14 at instant $t=6$, from an initial value of -20 at $t=0$. Figure 1 also shows the evolution of $F$ and $A L$ for the same simulation. Obviously, growth of expected fund assets and expected liabilities over time is observed, since benefits present a positive mean increase. This trend is seen in the next graph, where the expected values of debt, fund and actuarial liability are shown. These curves have been computed with Monte Carlo simulation, see e.g. Kloeden and Platen (1999). The expected value of $X$ is increasing, that is, the expected debt decreases. In our example, mean debt is reduced from -20 to -7.18 , that is, $64 \%$ of its value. This fact is better appreciated in the fourth graph, where $\mathbb{E} F(t)$ gets closer to $\mathbb{E} A L(t)$ as $t$ increases.

\section{[INSERT FIGURE 1 HERE]}

Figure 2 represents the proportions of the fund invested in the portfolio in order to minimize the terminal solvency risk. The paths correspond to the same sample as in Figure 1. These functions depend on the individual values of correlations $q_{1}$ and $q_{2}$, see (15) and (16), whereas the processes in Figure 1 depend on the aggregate value $q_{1}^{2}+q_{2}^{2}$, see (17). Thus we consider four possible scenarios: $\left(q_{1}, q_{2}\right)=(-0.2,-0.2),(-0.2,0.2)$,

\footnotetext{
${ }^{5}$ It should be possible to apply other methods with higher order of convergence, as the Milstein scheme, see e.g. Kloeden and Platen (1999). For our purposes it suffices the Euler scheme. The calculations have been done with MATLAB ${ }^{\circledR}$.
} 
$(0.2,-0.2),(0.2,0.2)$.

\section{[INSERT FIGURE 2 HERE]}

The four graphs in Figure 2 show a similar pattern. In the first years where debt is large, the optimal strategy is to take more risk, borrowing money to invest in the bond and in the stock. The higher mean returns they provide compared with the bank account is the factor that may explain this behavior. In fact, the time when debt takes the maximum value, that is, when $X$ is minimum, is just when $\lambda_{B}^{*}+\lambda_{S}^{*}$ also attains its maximum value. At this point the strategy is quite aggressive indeed, requiring the borrowing of money for the amount of approximately $278 \%$ of the fund's wealth, or $2.78 F$ to invest with risk (first graph in Figure 2, with negative value of both correlations). In the final part of the time interval the amount held in cash increases and the amount invested in the stock and the bond diminishes, considerably reducing the risky composition of the portfolio. The behavior described is similar in the four cases of correlations considered.

The relative weight of the stock and the bond in the portfolio is highly influenced by the signs of correlations, at least in the sample shown. In the first two graphs where $q_{1}<0$, the bond participates in the portfolio in a larger proportion than the stock, independently of the sign of $q_{2}$. When $q_{1}>0$ the situation is reversed, except the last year. Thus, the feature observed in the model studied in Menoncin (2005), where the bond's share in the portfolio is larger than the share of the stock, is not maintained in our model. This different behavior may be due, on the one hand, to the existence of correlations and on the other hand, to the aim of the sponsor to minimize the expected square of debt, instead of maximizing expected utility from surplus.

\section{Conclusions}

We have analyzed the management of a pension funding process of a DB pension plan when the short interest rate is the Vasicek model. The problem of the minimization of the terminal solvency risk has been solved analytically when the benefits process is a geometric Brownian motion under a suitable selection of the technical interest rate. The components of the optimal portfolio (investments in the bond, in the stock and in the 
cash) are the sum of two terms, one proportional to the unfunded actuarial liability, and another to the actuarial liability, depending on parameters of the randomness of benefits and its correlations with the interest rate and the stock.

We have done a numerical simulation showing some properties of the model. Though there are three sources of randomness, the debt is reduced by means of risky investment in the first years and with a more conservative investment policy in the last years of the planned period.

Further research should include other dynamics for the interest rate processes, such as the Cox-Ingersoll-Ross (CIR) model, the Ho-Lee model or affine models in general. As for benefits, it would also be interesting to consider the possibility of jumps, such as in Ngwira and Gerrard (2006), or some more general Lévy process.

\section{A Appendix}

Proof of Proposition 2.1. The process $\Delta_{t}(u)=e^{-\int_{t}^{u} \delta(s) d s}$ satisfies

$$
d \Delta_{t}(u)=-\delta(u) \Delta_{t}(u) d u, \quad \Delta_{t}(t)=1,
$$

hence, by Assumption A, $\Delta_{t} P$ is a geometric Brownian motion with non-constant coefficients satisfying

$$
d\left(\Delta_{t} P\right)(u)=\Delta_{t}(u) d P(u)+d \Delta_{t}(u) P(u)=\left(\Delta_{t} P\right)(u)\left((\mu-\delta(u)) d u+\eta d w_{P}(u)\right) .
$$

This follows from the integration by parts formula since $d \Delta_{t}$ has no diffusion term, see e.g. Karatzas and Shreve (1997). Then, the conditional expectation is

$$
\mathbb{E}\left(\Delta_{t}(t+d-x) P(t+d-x) \mid \mathcal{F}_{t}\right)=\Delta_{t}(t) P(t) e^{\int_{t}^{t+d-x}(\mu-\delta(u)) d u},
$$

thus, recalling the definition of $A L$ and $\psi_{A L}$ we get:

$$
A L(t)=P(t) \int_{a}^{d} e^{\int_{t}^{t+d-x}(\mu-\delta(u)) d u} M(x) d x=P(t) \psi_{A L}(t)
$$

because $\Delta_{t}(t)=1$. Analogously, $N C(t)=P(t) \psi_{N C}(t)$. 
Now, by means an integration by parts, and the definition of $\xi_{A L}$ we have

$$
\begin{aligned}
\psi_{N C}(t) & =\int_{a}^{d} e^{\int_{t}^{t+d-x}(\mu-\delta(s)) d s} d M(x) \\
& =\left.e^{\int_{t}^{t+d-x}(\mu-\delta(s)) d s} M(x)\right|_{x=a} ^{x=d}+\int_{a}^{d} e^{\int_{t}^{t+d-x}(\mu-\delta(s)) d s}(\mu-\delta(t+d-x)) M(x) d x \\
& =1+\xi_{A L}(t)+(\mu-\delta(t)) \psi_{A L}(t) .
\end{aligned}
$$

In consequence

$$
\begin{aligned}
N C(t) & =\psi_{N C}(t) P(t) \\
& =P(t)+\xi_{A L}(t) P(t)+(\mu-\delta(t)) \psi_{A L}(t) P(t) \\
& =P(t)+\left(\mu-\delta(t)+\frac{\xi_{A L}(t)}{\psi_{A L}(t)}\right) A L(t),
\end{aligned}
$$

which is (1). Finally we deduce the stochastic differential equation that the actuarial liability satisfies. Notice that $d \psi_{A L}(t)=\xi_{A L}(t) d t$. Thus, using Assumption A,

$$
\begin{aligned}
d A L(t) & =d\left(\psi_{A L} P\right)(t) \\
& =\psi_{A L}(t) d P(t)+d \psi_{A L} P(t) \\
& =\psi_{A L}(t) P(t)\left(\mu d t+\eta d w_{P}(t)\right)+\xi_{A L}(t) P(t) d t \\
& =\left(\mu+\frac{\xi_{A L}(t)}{\psi_{A L}(t)}\right) A L(t) d t+\eta A L(t) d w_{P}(t),
\end{aligned}
$$

with the initial condition $A L(0)=A L_{0}=\psi_{A L}(0) P_{0}$.

Proof of Theorem 5.1. Consider the value function (14) of the control problem (2), (4), (12), (13). This function so defined is non-negative and strictly convex. Under some sufficient conditions, including smoothness, $\widehat{V}$ is a solution of the HJB equation, see Fleming and Soner (1993):

$$
\begin{aligned}
V_{t}+\min _{\lambda_{B}, \lambda_{S}}\{ & \left(b \sigma \zeta \lambda_{B}+m_{S} \lambda_{S}+(r-k) X+(r-\delta) A L\right) V_{X}+\left(\mu+\xi_{A L} / \psi_{A L}\right) A L V_{A L}+\alpha(\beta-r) V_{r} \\
& +\frac{1}{2}\left(\left(1-q_{1}^{2}-q_{2}^{2}\right) \eta^{2} A L^{2}+\left(b \sigma \lambda_{B}-\sigma_{r} \lambda_{S}+\eta q_{1} A L\right)^{2}+\left(\sigma_{S} \lambda_{S}-\eta q_{2} A L\right)^{2}\right) V_{X X} \\
& +\frac{1}{2} \eta^{2} A L^{2} V_{A L, A L}+\left(-\eta^{2} A L^{2}+\eta q_{1}\left(\sigma_{r} \lambda_{S}-b \sigma \lambda_{B}\right) A L+\eta q_{2} \sigma_{S} \lambda_{S} A L\right) V_{X, A L} \\
& \left.+\frac{1}{2} \sigma^{2} V_{r r}+\sigma \eta q_{1} A L V_{r, A L}+\sigma\left(-b \sigma \lambda_{B}+\sigma_{r} \lambda_{S}-\eta q_{1} A L\right) V_{r X}\right\}=0,
\end{aligned}
$$




$$
V(T, X, A L, r)=X^{2}
$$

If there exists a smooth solution $V$ of this equation, strictly convex with respect to $X$, then the optimal values of the investments are given by

$$
\begin{aligned}
& \widehat{\lambda_{B}}\left(V_{X}, V_{X X}, V_{X, A L}, V_{r X}\right)=\frac{1}{b \sigma \sigma_{S}^{2} V_{X X}}(-\left(\zeta\left(\sigma_{r}^{2}+\sigma_{S}^{2}\right)+m_{S} \sigma_{r}\right) V_{X}+\sigma \sigma_{S}^{2} V_{r X} \\
&\left.+\eta \sigma_{S}\left(\sigma_{r} q_{2}-\sigma_{S} q_{1}\right) A L\left(V_{X X}-V_{X, A L}\right)\right), \\
& \widehat{\lambda_{S}}\left(V_{X}, V_{X X}, V_{X, A L}\right)=\frac{1}{\sigma_{S}^{2} V_{X X}}\left(-\left(m_{S}+\zeta \sigma_{r}\right) V_{X}+\eta \sigma_{S} q_{2} A L\left(V_{X X}-V_{X, A L}\right)\right) .
\end{aligned}
$$

After substitution of these values in (18) we obtain that $\widehat{V}$ satisfies

$$
\begin{aligned}
V_{t} & +\left((r-k) X+(r-\delta) A L+\frac{\eta}{\sigma_{S}}\left(\zeta\left(q_{2} \sigma_{r}+q_{1} \sigma_{S}\right)+m_{S} q_{2}\right) A L\right) V_{X} \\
& +\left(\mu+\frac{\xi_{A L}}{\psi_{A L}}\right) A L V_{A L}+\alpha(\beta-r) V_{r} \\
& +\frac{1}{2}\left(1-q_{1}^{2}-q_{2}^{2}\right) \eta^{2} A L^{2} V_{X X}+\frac{1}{2} \eta^{2} A L^{2} V_{A L, A L}+\frac{1}{2} \sigma^{2} V_{r r} \\
& -\left(1-q_{1}^{2}-q_{2}^{2}\right) \eta^{2} A L^{2} V_{X, A L}+\eta q_{1} \sigma A L V_{r, A L} \\
& -\frac{1}{2 \sigma_{S}^{2}}\left(\left(m_{S}+\zeta \sigma_{r}\right)^{2}+\zeta^{2} \sigma_{S}^{2}\right) \frac{V_{X}^{2}}{V_{X X}}+\zeta \eta q_{1} A L \frac{V_{X} V_{X, A L}}{V_{X X}}+\zeta \sigma \frac{V_{X} V_{r X}}{V_{X X}} \\
& -\frac{1}{2} \sigma^{2} \frac{V_{r X}^{2}}{V_{X X}}-\frac{1}{2} \eta^{2}\left(q_{1}^{2}+q_{2}^{2}\right) A L^{2} \frac{V_{X, A L}^{2}}{V_{X X}}-\eta q_{1} \sigma A L \frac{V_{r X} V_{X, A L}}{V_{X X}}=0,
\end{aligned}
$$

with the final condition (19). We will use a guessing method ${ }^{6}$ to solve (22), trying a quadratic solution of the form

$$
\widehat{V}(t, X, A L, r)=f_{X X}(t, r) X^{2}+f_{A L, A L}(t, r) A L^{2}+f_{X, A L}(t, r) X A L,
$$

\footnotetext{
${ }^{6}$ Once a smooth solution of the PDE and the final condition is found, further conditions are needed to check in order to be sure that actually it is the value function. They are existence and uniqueness of a strong solution of the optimal SDEs (2), (4) and (17), and admissibility of the controls $\hat{\lambda}_{B}, \widehat{\lambda}_{S}$ in the sense of (9). In our model this conditions are fulfilled, since the controls turn out to be linear in $X$. Thus, the SDEs can be reduced to a single one in process $X$-linear, with stochastic coefficients-, once the explicit expressions for $r$ and $A L$ are substituted into (17).
} 
and the following ordinary differential equations are obtained for the above coefficients:

$$
\begin{aligned}
\left(f_{X X}\right)_{t} & +\left(-\zeta^{2}-\frac{\left(m_{S}+\zeta \sigma_{r}\right)^{2}}{\sigma_{S}^{2}}+2(r-k)\right) f_{X X}+(2 \zeta \sigma+\alpha(\beta-r))\left(f_{X X}\right)_{r} \\
& -\sigma^{2} \frac{\left(f_{X X}\right)_{r}^{2}}{f_{X X}}+\frac{\sigma^{2}}{2}\left(f_{X X}\right)_{r r}=0, \quad f_{X X}(T, r)=1 . \\
\left(f_{A L, A L}\right)_{t}- & \frac{1}{4}\left(\zeta^{2}+\frac{\left(m_{S}+\zeta \sigma_{r}\right)^{2}}{\sigma_{S}^{2}}-2 \zeta \eta q_{1}+\eta^{2}\left(q_{1}^{2}+q_{2}^{2}\right)\right) \frac{f_{X, A L}^{2}}{f_{X X}}+2\left(\mu+\xi_{A L} / \psi_{A L}\right) f_{A L, A L} \\
+ & \left(r-\delta-\zeta \eta q_{1}+\eta q_{2} \frac{m_{S}+\zeta \sigma_{r}}{\sigma_{S}}-\left(1-\left(q_{1}^{2}+q_{2}^{2}\right)\right) \eta^{2}\right) f_{X, A L}+\frac{\sigma}{2}\left(\zeta-\eta q_{1}\right) \frac{f_{X, A L}\left(f_{X, A L}\right)_{r}}{f_{X X}} \\
+ & \alpha(\beta-r)\left(f_{A L, A L}\right)_{r}+\left(1-q_{1}^{2}-q_{2}^{2}\right) \eta^{2} f_{X X}-\frac{\sigma^{2}}{4} \frac{\left(f_{X, A L}\right)_{r}^{2}}{f_{X X}}+\eta^{2} f_{A L, A L}+\frac{\sigma^{2}}{2}\left(f_{A L, A L}\right)_{r r} \\
+ & 2 \eta q_{1} \sigma\left(f_{A L, A L}\right)_{r}=0, \quad f_{A L, A L}(T, r)=0 . \\
\left(f_{X, A L}\right)_{t}+ & \left(-\zeta^{2}-\frac{\left(m_{S}+\zeta \sigma_{r}\right)^{2}}{\sigma_{S}^{2}}+\zeta \eta q_{1}+r-k+\mu+\frac{\xi_{A L}}{\psi_{A L}}\right) f_{X, A L}+\frac{\sigma^{2}}{2}\left(f_{X, A L}\right)_{r r} \\
& +2\left(r-\delta-\zeta \eta q_{1}+\eta q_{2} \frac{m_{S}+\zeta \sigma_{r}}{\sigma_{S}}\right) f_{X X}+\left(\zeta \sigma+\eta q_{1} \sigma+\alpha(\beta-r)\right)\left(f_{X, A L}\right)_{r} \\
+ & \left(\zeta-\eta q_{1}\right) \sigma \frac{f_{X, A L}\left(f_{X X}\right)_{r}}{f_{X X}}-\sigma^{2} \frac{\left(f_{X, A L}\right)_{r}\left(f_{X X}\right)_{r}}{f_{X X}}=0, \quad f_{X, A L}(T, r)=0 .
\end{aligned}
$$

In order to solve $(24)$, we try $f_{X X}(t, r)=g(t) e^{\gamma(t) r}$, with the final conditions $g(T)=1$ and $\gamma(T)=0$, and after simplification we obtain

$$
\dot{g}+(\dot{\gamma}-\alpha \gamma+2) r g+\left(-\frac{\sigma^{2}}{2} \gamma^{2}+(2 \zeta \sigma+\alpha \beta) \gamma-2 k-\zeta^{2}-\frac{\left(m_{S}+\zeta \sigma_{r}\right)^{2}}{\sigma_{S}^{2}}\right) g=0 .
$$

Choosing $\gamma$, such that $\dot{\gamma}-\alpha \gamma+2=0$, function $g$ is given by $\dot{g}+h g=0$, where

$$
h(t)=-\left(\sigma^{2} / 2\right) \gamma^{2}(t)+(2 \zeta \sigma+\alpha \beta) \gamma(t)-2 k-\zeta^{2}-\left(m_{S}+\zeta \sigma_{r}\right)^{2} / \sigma_{S}^{2}
$$

With the final conditions we obtain

$$
\gamma(t)=\frac{2}{\alpha}\left(1-e^{-\alpha(T-t)}\right)
$$

and $g(t)=e^{H(T)-H(t)}$ with $H$ a primitive of $h$. Hence we obtain

$$
f_{X X}(t, r)=e^{H(T)-H(t)+(2 / \alpha)\left(1-e^{-\alpha(T-t)}\right) r} .
$$


Using Assumption C, it is easy to prove that function $f_{X, A L}$, satisfying (25), is $f_{X, A L}=0$. Inserting (23) into (20)-(21) we obtain that the optimal investments are given by

$$
\begin{aligned}
\lambda_{B}^{*}(t, X, A L, r)= & \frac{1}{b}\left(-\frac{\zeta\left(\sigma_{r}^{2}+\sigma_{S}^{2}\right)+m_{S} \sigma_{r}}{\sigma \sigma_{S}^{2}}+\frac{\left(f_{X X}\right)_{r}}{f_{X X}}\right) X \\
& +\frac{1}{2 b f_{X X}}\left(-\frac{\zeta\left(\sigma_{r}^{2}+\sigma_{S}^{2}\right)+m_{S} \sigma_{r}}{\sigma \sigma_{S}^{2}} f_{X, A L}+\left(f_{X, A L}\right)_{r}\right) A L \\
& +\frac{\eta\left(\sigma_{r} q_{2}-\sigma_{S} q_{1}\right)}{b \sigma \sigma_{S}}\left(1-\frac{f_{X, A L}}{2 f_{X X}}\right) A L \\
\lambda_{S}^{*}(t, X, A L, r)= & -\frac{m_{S}+\sigma_{r} \zeta}{\sigma_{S}^{2}} X+\frac{\eta q_{2}}{\sigma_{S}} A L,
\end{aligned}
$$

that is to say, (15) and (16), respectively, because $f_{X X}(t, r)=g(t) e^{\gamma(t) r}$ and $f_{X, A L}=0$. 


\section{References}

Battocchio, P, Menoncin, F., 2004. Optimal pension management in a stochastic framework. Insurance, Mathematics and Economics 34, 79-95.

Boulier, J.F., Huang, S., Taillard, G., 2001. Optimal management under stochastic interest rates: the case of a protected defined contribution pension fund. Insurance: Mathematics and Economics 28, 173-189.

Bowers, N.L., Gerber, H.U., Hickman, J.C., Jones, D.A., Nesbitt, C.J., 1986. Actuarial Mathematics. The Society of Actuaries, Itaca.

Cairns, A.J.G., Blake, D., Dowd, K., 2006. Stochastic lifestyling: Optimal dynamic asset allocation for defined contribution pension plans. Journal of Economic Dynamics and Control 30, 843-877.

Constantinides, G., 1978. Market risk adjustment and project valuation. Journal of Finance 33, 603-616.

Chang, S.-C., 1999. Optimal pension funding through dynamic simulations: the case of Taiwan public employees retirement system. Insurance: Mathematics and Economics 24, $187-199$.

Chang, S.-C., Tzeng, L.Y., Miao, J.C.Y., 2003. Pension funding incorporating downside risks. Insurance: Mathematics and Economics 32, 217-228.

Deelstra, G., Grasselli, M., Koehl, P.-F., 2003. Optimal investment strategies in the presence of a minimum guarantee. Insurance, Mathematics and Economics 33, 189-207. Duffie, D., Kan, R., 1996. A yield-factor model of interest rates. Mathematical Finance 6, 379-406.

Fleming, W.H., Soner, H.M., 1993. Controlled Markov Processes and Viscosity Solutions. Springer Verlag, New York.

Haberman, S., Butt, Z., Megaloudi, C., 2000. Contribution and solvency risk in a defined benefit pension scheme. Insurance: Mathematics and Economics 27, 237-259.

Haberman, S., Sung, J.H., 1994. Dynamics approaches to pension funding. Insurance: Mathematics and Economics 15, 151-162.

Haberman, S., Vigna, E., 2002. Optimal investment strategies and risk measures in defined contribution pension schemes. Insurance: Mathematics and Economics 31, 3569.

Josa-Fombellida, R., Rincón-Zapatero, J.P., 2001. Minimization of risks in pension fund- 
ing by means of contribution and portfolio selection. Insurance: Mathematics and Economics 29, 35-45.

Josa-Fombellida, R., Rincón-Zapatero, J.P., 2004. Optimal risk management in defined benefit stochastic pension funds. Insurance: Mathematics and Economics 34, 489-503.

Josa-Fombellida, R., Rincón-Zapatero, J.P., 2006. Optimal investment decisions with a liability: The case of defined benefit pension plans. Insurance: Mathematics and Economics 39, 81-98.

Josa-Fombellida, R., Rincón-Zapatero, J.P., 2008a. Funding and investment decisions in a stochastic defined benefit pension plan with several levels of labor-income earnings. Computers and Operations Research 35, 47-63.

Josa-Fombellida, R., Rincón-Zapatero, J.P., 2008b. Mean-variance portfolio and contribution selection in stochastic pension funding. European Journal of Operational Research 187, 120-137.

Karatzas, I., Shreve, S.E., 1997. Brownian Motion and Stochastic Calculus. Springer, Berlin.

Kloeden, P.E., Platen, E., 1999. Numerical Solution of Stochastic Differential Equations. Springer, Berlin.

Menoncin, F., 2005. Cyclical risk exposure of pension funds: A theoretical framework. Insurance, Mathematics and Economics 36, 469-484.

Merton, R.C., 1971. Optimal consumption and portfolio rules in a continuous-time model. Journal of Economic Theory 3, 373-413.

Ngwira, B., Gerrard, R., 2006. Stochastic pension fund control in the presence of Poisson jumps. Insurance: Mathematics and Economics 40, 283-292.

Owadally, M.I., Haberman, S., 1999. Pension fund dynamics and gains/losses due to random rates of investment return. North American Actuarial Journal 3, 105-117.

Øksendal, B., 2003. Stochastic Differential Equations. Springer, Berlin.

Vaşicek, O., 1977. An equilibrium characterisation of the term structure. Journal of Financial Economics 5, 177-188.

Vigna, E., Haberman, S., 2001. Optimal investment strategy for defined contribution pension schemes. Insurance: Mathematics and Economics 28, 233-262. 
Table 1

Values of parameters

Interest rate

Mean reversion, $\alpha \quad 0.2$

Mean rate, $\beta \quad 0.05$

Volatility, $\sigma \quad 0.02$

Initial rate, $r_{0} \quad 0.05$

Maturity bond

Maturity, $T_{1} \quad 10$

Market price of risk, $\zeta \quad 0.15$

Stock

Risk premium, $m_{S} \quad 0.06$

Interest rate source risk, $\sigma_{r} \quad 0.06$

Stock own volatility, $\sigma_{S} \quad 0.19$ 

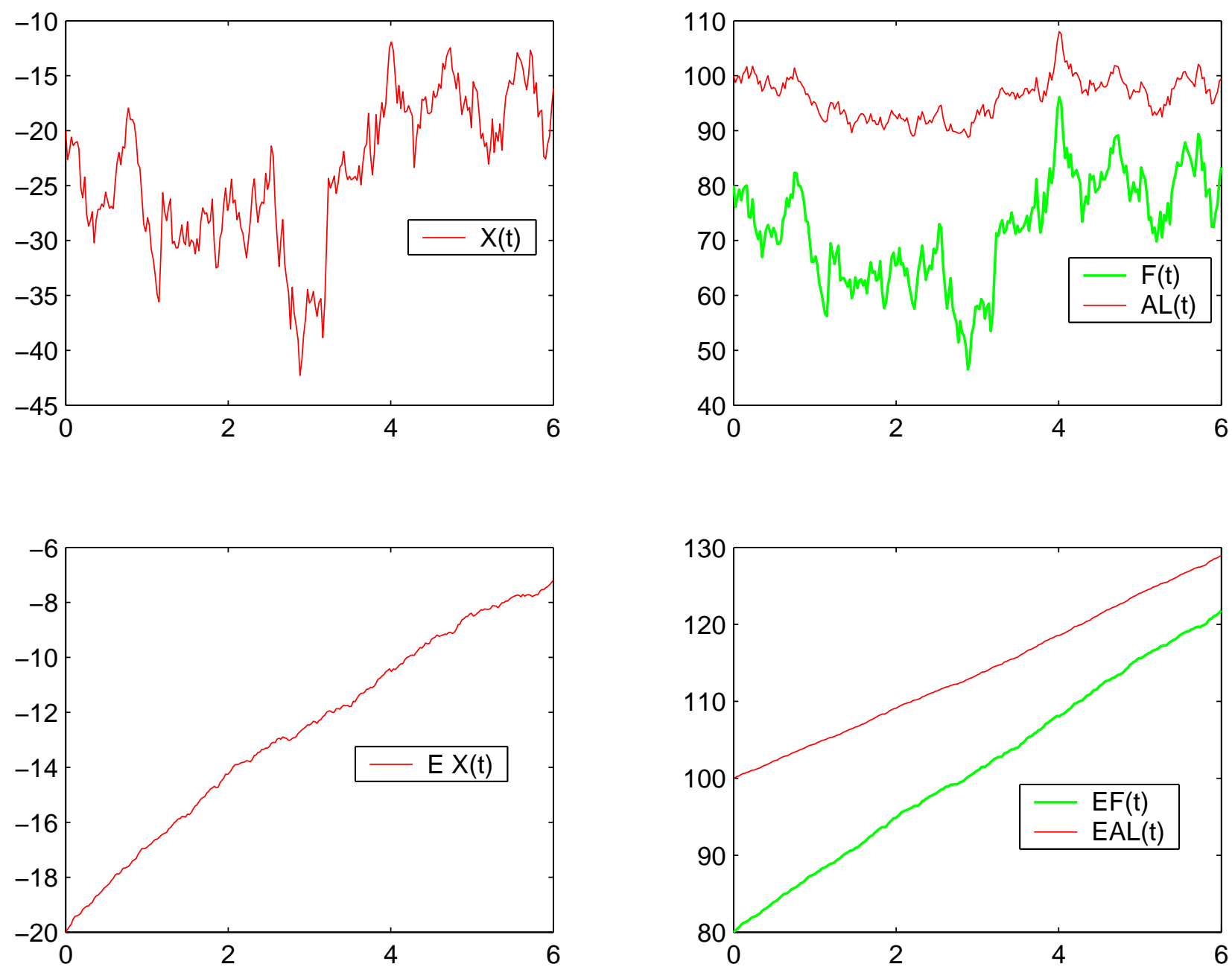

Fig. 1. Debt, fund, actuarial liability and their expected values. 

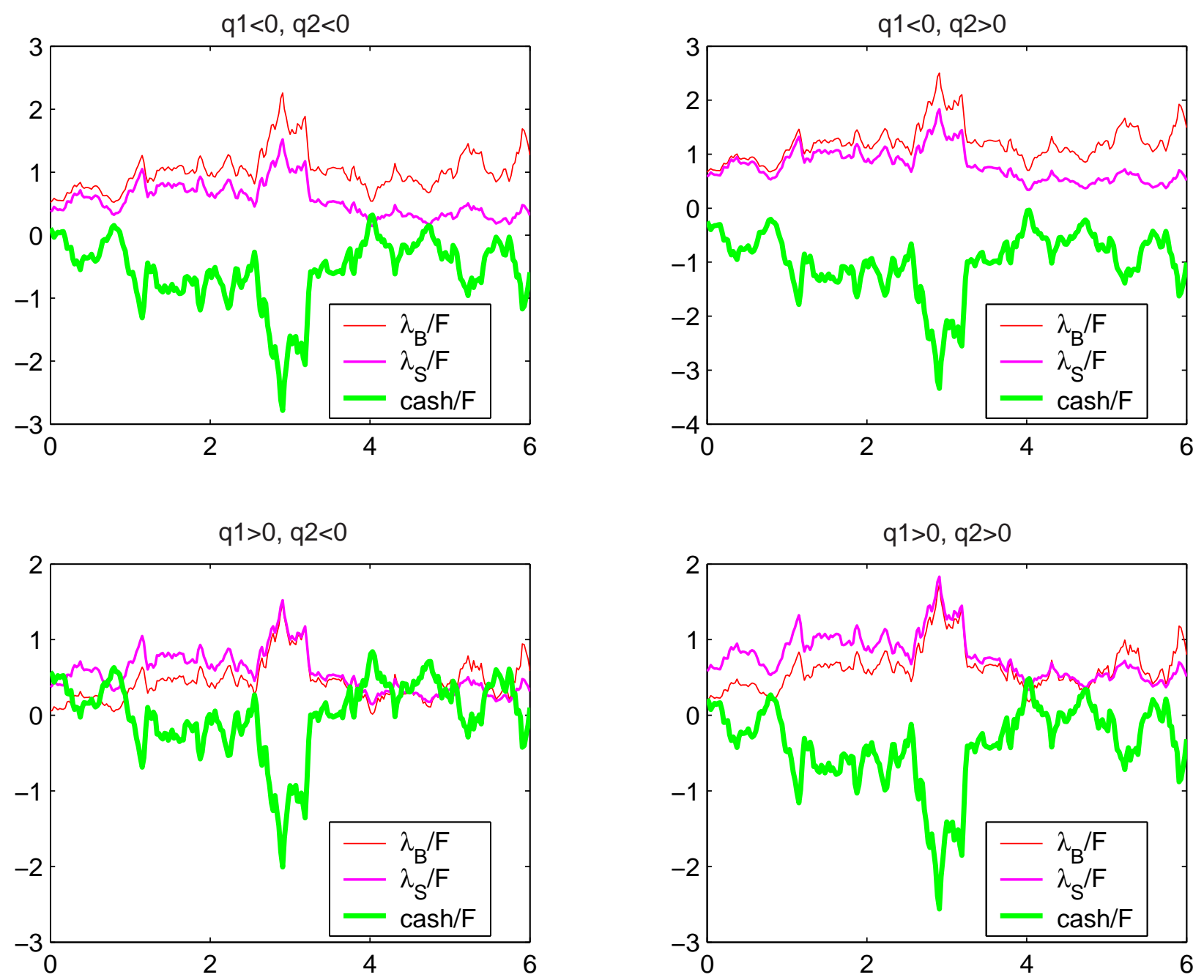

Fig. 2. Investment proportions for four cases of correlations. 